\title{
Reward structure and payoff preferences in human learning'
}

\author{
MICHAEL COLE, YALE UNIVERSITY
}

ROBERT CALFEE, UNIVERSITY OF WISCONSIN
LEO KELLER, STANFORD UNIVERSITY

Ss' preferences when choosing between a pair of token rewards, one a known and the other an unknown value, were determined under two conditions, (1) the range of possible reward values was known from the outset of the experiment and (2) the range had to be learned. Analysis of choice probabilities and latencies indicated choices yielded almost optimal outcomes although there was some tendency to choose a non-optimal unknown alternative if it might possibly yield a large reward. Choices of known alternatives closer to the endpoints of the range of values were made more quickly even though choice probabilities were equal, and choices were made more slowly by $S s$ who had to learn the range of values.

A number of studies in recent years have sought to determine the role of rewards in human learning and decision making (Myers \& Atkinson, 1964; Keller, Cole, Burke, \& Estes, 1964; Katz, 1964). In some studies, reward has been manipulated by paying Ss small sums of money following each trial, typically in a range of one to ten cents. In other experiments, poker chips, tokens or points, indicating "how well you are doing" have been used as reinforcements. Very little data are available on Ss' relative preferences for these token rewards. In general, a monotonic preference scale has been assumed, since it seems reasonable that normal human Ss will prefer three points over two points, etc. Although this assumption may be adequate for certain types of investigations, detailed quantitative studies of reward variables require more information about preferences for various amounts of surrogate rewards. In the present study, S was required to perform a modified pairedcomparison task in which, on each trial, a choice was made between two token payoff values. One value was visible in the stimulus display, while the other alternative was represented by an " $\mathrm{X}$ ' until a choice was made. To obtain information about the effect of prior knowledge of the range of point values, one group of Ss was told in advance what the range was, while a second group was given no information.

\section{Method}

Subjects. The Ss were 40 introductory psychology students at Stanford University in the spring quarter ${ }_{\diamond} 1964$. Participation satisfied a course requirement, and Ss did not receive any payment for participation.

Apparatus. The $\mathrm{S}$ was seated at a table on which there was a response panel with two buttons. The button to the right was labelled "A," that to the left "X." Centered below the two buttons was a handrest on which $\mathrm{S}$ was required to place his hand between responses. Five $\mathrm{ft}$. in front of the table were two visual display units, one placed on top of the other. Each unit had a 2 in. $x$ 12 in. screen on which numeric characters $(2$ in. $x$ 1-1/2 in.) could be presented. Presentation of the stimuli, control of time intervals, as well as recording of the choice response and latency were controlled by an IBM card punch and associated equipment located in an adjacent room. Latencies were measured to the nearest .01 sec. by a Beckman timer.

Procedure. Each trial began with the presentation in the upper display unit of the stimulus for that trial. The stimulus was always of the form "A-X," where " $A$ " was one of the numbers $1,2,4,6$, or 8 . The $S$ then pushed the button corresponding to his choice for that trial; an "A" response indicated choice of the known point value displayed, while an " $X$ " response indicated choice of the unknown point value. Following the response, the pair of actual values for that trial was presented in the lower display unit for a 2 sec. reinforcement interval. The response button lit up when pressed and remained on during the reinforcement interval so that $S$ was able to compare the payoff values with his actual choice. The two displays were then turned off, and 2 sec. later the next stimulus pair was presented. For example, on a given trial the stimulus display might be "4-X," and after his choice, S might be presented with the reinforcement pair "4-6." Choice of "A" on this trial would result in a 4-point reward, while the choice of " $\mathrm{X}$ " would yield a 6-point reward.

There were 10 blocks of 25 trials; in each block each pairwise combination of the numbers $1,2,4,6$, and 8 in position " $\mathrm{A}$ " and the same set of numbers in position " $X$ " occurred exactly once. The presentation order of the 25 pairwise combinations was separately randomized within each block for each S.

The two groups differed only in the information they received concerning the range of point values involved.

The "known range" (KR) group was told,

"On each trial of the experiment a number will light up on the upper screen in front of you. This number will be $1,2,4,6$, or 8 . Next to the number will be an $X$. The $X$ on the screen represents another number.It will also be one of the numbers $1,2,4,6$, or 8 . On each trial you must try to choose the number which will give you the largest payoff."

The "unknown range" (UR) group was given the same instructions but with the two italicized sentences deleted. 




Fig. 1. Proportion of optimum choices of each payoff value in blocks of 25 trials (5 trials per value) for known Range and Unknown Range groups.

\section{Results and Discussion}

Figure 1 presents the proportion of optimum choices in each block of five trials for each payoff condition. A choice was considered optimum if it yielded the largest expected gain. Thus, choosing 8 and 6 was optimum when $8-X$ and $6-X$ were presented, while choosing $X$ was optimum when $4-\mathrm{X}, 2-\mathrm{X}$, and $1-\mathrm{X}$ were presented. The value 4 is not quite a midpoint in the series. In a single cycle through the list, the payoff from $4-X$ items if 4 were always chosen would be 20 points, while the payoff for always choosing $X$ would be 21 points.

From Fig. 1 it may be seen that $S s$ in the $K R$ group quickly settled on the optimum choice for 1-X, 2-X, and 8-X. The proportion of optimum choices for the 6-X items was consistently below the levels attained by the above items. The proportion of choices of $X$ for 4-X was above .5 , but distinctly below all other items. The mean proportions for the UR group were lower for all choices during the first block of five trials, but during subsequent blocks, the means were close to the values of the KR group.

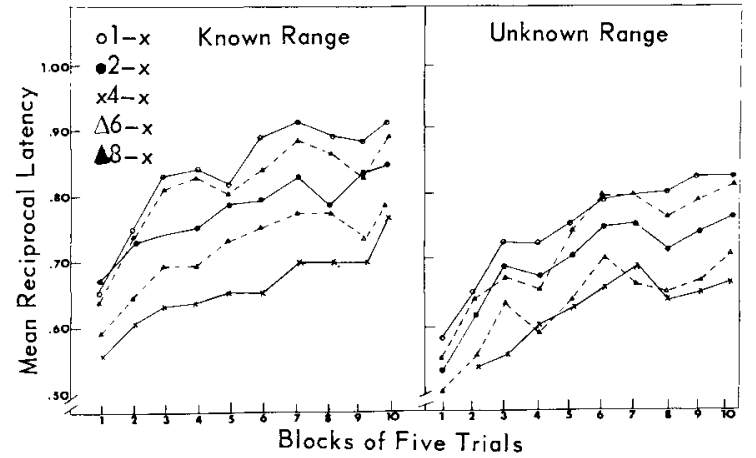

Fig. 2. Mean response speeds for each payoff value in blocks of 25 trials for (5 trials per value) Known Range and Unknown Range groups.
A somewhat different picture emerges from analysis of response speeds for the different items (Fig. 2). Speeds for both groups reveal a clear ordering

$$
(1-\mathrm{X})<(8-\mathrm{X})<(2-\mathrm{X})<(6-\mathrm{X})<(4-\mathrm{X}) \text {. }
$$

The ordering may be summarized as follows: (1) response speeds were higher the further an item was from the midpoint of the scale and (2) for pairs of items located symmetrically about $4-X$ (1-X vs. $8-X$, and $2-X$ vs. $6-\mathrm{X})$, items with the smaller known values had shorter latencies. In addition, the overall choice latencies of the KR group were consistently lower than those of the UR group. An analysis of variance of the response times using a reciprocal transformation indicated that the difference between the two groups approached significance, $\mathrm{F}=3.15, \mathrm{df}=1 / 38, \mathrm{p}<.10$. Speed varied significantly with items, $F=53.9, \mathrm{df}=4 / 152, \mathrm{p}<.01$, and trials, $F=23.0, \mathrm{df}=9 / 342, \mathrm{p}<.01$, and no other sources of variance were significant.

The choice data in Fig. 1 lend support to the statement that Ss dichotomize the range of payoffs according to a rational scheme. Instructions may be used to set the range immediately, and thus speed up the dichotomizing process, but the range of values was quickly learned even in the absence of information. However, certain deviations from this scheme should be mentioned. Even in the $\mathrm{KR}$ group, the $2-\mathrm{X}$ and $6-\mathrm{X}$ items are not quite symmetric. Given $6-\mathrm{X}, \mathrm{S}$ will occasionally attempt to obtain the 8-point payoff. This asymmetry is accentuated in the UR group. The $\mathrm{X}$ or unknown payoff is also more attractive to the UR group when responding to 4-X. Asymmetries also appear in the response time data (Fig. 2) in that Ss respond more slowly at the upper end of the payoff range than they do for an equivalent item at the lower end of the range. It appears that even for Ss who know the range, the lower end of the scale is anchored by the zero point whereas the upper end of the range is relatively free. The slower responding of the UR group may also reflect uncertainty about the range of payoffs. The fact that noticeable differences in choice times persist when choice probabilities have reached common values may be viewed as support to the notion that the strength of approach and avoidance tendencies may be relatively independent of overt choice behavior (cf. Keller et al, 1965).

\section{References}

Katz, L. Effects of differential monetary gain and loss on sequential two-choice behavior. J. exp. Psychol., 1964, 67, 245-249.

Keller, L., Cole, M., Burke, C. J., \& Estes, W. K. Reward and information values of trial outcomes in paired-associate learning. Psychol. Monogr., 1965, 79, No. 12 (Whole No. 605).

Myers, J. L., \& Atkinson, R. C. Choice behavior and reward structure. J. math. Psychol., 1964, 1, 170-203.

\section{Note}

1. This work was carried out at the Institute for Mathematical Studies in the Social Sciences, Stanford University. Thanks are due to W. K. Estes who contributed the apparatus used in the study. 